\title{
Experimental study of the thermodynamics of an interacting trapped Bose-Einstein condensed gas
}

\author{
F. Gerbier, ${ }^{1, *}$ J. H. Thywissen, ${ }^{1,2}$ S. Richard, ${ }^{1}$ M. Hugbart, ${ }^{1}$ P. Bouyer, ${ }^{1}$ and A. Aspect ${ }^{1}$ \\ ${ }^{1}$ Groupe d'Optique Atomique, Laboratoire Charles Fabry de l'Institut d'Optique, 91403 Orsay Cedex, France \\ ${ }^{2}$ Department of Physics, University of Toronto, Toronto, Ontario, M5S 1A7, Canada
}

(Received 28 January 2004; published 19 July 2004)

\begin{abstract}
We have investigated experimentally the finite-temperature properties of a Bose-Einstein condensed cloud of ${ }^{87} \mathrm{Rb}$ atoms in a harmonic trap. Focusing primarily on condensed fraction and expansion energy, we measure unambiguous deviations from ideal-gas thermodynamics and obtain good agreement with a Hartree-Fock description of the mixed cloud. Our results offer clear evidence of the mutual interaction between the condensed and thermal components. To probe the low-temperature region unaccessible to the usual time-of-flight technique, we use coherent Bragg scattering as a filtering technique for the condensate. This allows us to separate spatially the condensed and normal components in time of flight and to measure reliably temperatures as low as $0.2 T_{c}^{0}$ and thermal fractions as low as $10 \%$. Finally, we observe evidence for the limitations of the usual image analysis procedure, pointing out to the need for a more elaborate model of the expansion of the mixed cloud.
\end{abstract}

DOI: 10.1103/PhysRevA.70.013607

PACS number(s): 03.75.Hh

\section{INTRODUCTION}

Trapped, dilute atomic gases offer an opportunity to study the interplay between quantum-statistical phenomena and interactions in Bose systems [1]. A third ingredient, the external trapping potential, also plays a key role in understanding the properties of these gases [2]. At finite temperatures, it leads to spatially distinct condensed and thermal phases, a new behavior when compared to bulk quantum fluids, in which both components overlap everywhere. This spatial separation allows in particular a clear identification of the condensed fraction through absorption imaging, in stark contrast with superfluid ${ }^{4} \mathrm{He}$, where condensed fraction measurements are only indirect [3].

Much work has been devoted to the properties of condensed gases at very low temperatures (much smaller than the critical temperature $T_{c}$ ), where the noncondensed fraction is negligible. Then, in the so-called Thomas-Fermi (TF) regime [2], the static and dynamic behavior of the condensate is essentially determined by the interplay between the trapping potential and the atomic interactions. At higher temperatures, less than but comparable to $T_{c}$, a significant thermal component is also present, typically much more dilute than the condensate. In this situation, the kinetic energy per thermal atom is larger than the mean-field energy, and deviations from ideal gas behavior are small [4-9]. Although several key thermodynamical properties, such as condensed fraction and average energy, are readily measurable experimentally, a detailed comparison of experiments with finitetemperature theories of the interacting cloud is to our knowledge still lacking.

The goal of this paper is to contribute to fill this gap by investigating experimentally the thermodynamics of a

\footnotetext{
*Present address: Institut für Physik, Johannes GutenbergUniversität, 55128 Mainz, Germany. Electronic address: gerbier@uni-mainz.de
}

trapped ${ }^{87} \mathrm{Rb}$ Bose gas below $T_{c}$, where both a condensed and a thermal component are present. We focus on two quantities, the condensed fraction and the expansion (kinetic plus mean-field) energy in the radial direction. We find that the condensed fraction $N_{0} / N$ is significantly reduced with respect to the ideal-gas law, $N_{0} / N=1-\left(T / T_{c}^{0}\right)^{3}$, where $T_{c}^{0}$ is the ideal-gas condensation temperature in the thermodynamic limit, and that the expansion energy is increased, even for the thermal atoms.

Our measurements thus clearly exclude ideal-gas behavior, and to assess the importance of interactions, we compare them with two mean-field theories of the interacting cloud that assume a condensate in the TF regime. The simplest one is the so-called "semi-ideal" model $[10,11]$, which considers the thermal cloud as a quantum-saturated gas evolving in the combined trapping plus condensate-mean-field potential. Although the general trend of our observations is well reproduced by this model, finer details are not. To take interactions within the thermal cloud and between the thermal cloud and the condensate into account, we use a self-consistent Hartree-Fock (HF) description of the mixed cloud [12-18], which yields good agreement with the data. We are able to confirm experimentally its validity over a wide range of temperatures and atom numbers, and to show that despite the diluteness of the thermal cloud, its mean-field energy affects both the condensed and noncondensed atoms. Such systematic measurements of the mutual interplay between the condensed and noncondensed components have not been reported before, although evidence for repulsion of the thermal atoms by the condensate has been provided in [19], through a careful study of the interface region between the two components.

Our measurements rely on the standard time-of-flight technique to observe the mixed cloud. A limitation arises at low temperatures, where the condensate appears broader than the thermal cloud in time of flight. This limits our measurements with this technique to $T / T_{c}^{0}>0.3$ (condensed fraction 
$<80 \%$ ). To overcome this limitation, we use coherent Bragg scattering to spatially separate the coherent and incoherent components of the cloud in time of flight. We apply it for low-temperature thermometry and measure in this way condensed fraction larger than $90 \%$ and temperatures below 100 $\mathrm{nK}\left(T / T_{c}^{0} \approx 0.2\right)$.

The paper is organized as follows. Section II first describes the experimental apparatus used for condensate production. Then, after reviewing the widely used procedure of time-of-flight imaging, we introduce the improved method based on Bragg diffraction to filter the condensate out of thermal cloud. The key results of this paper are presented in Secs. III and IV. In Sec. III, we present a measurement of the temperature dependence of the condensed fraction. In Sec. IV, the Bragg filtering scheme is applied to a measurement of the expansion energy of the thermal component. Both measurements clearly confirm the HF predictions in the whole temperature range. Further evidence for mutual interaction is provided in Sec. V, where we analyze the behavior of the mixed cloud in time of flight and measure a compression of the condensate axial length by the thermal cloud. For completeness, we review briefly in the Appendix I the three wellunderstood models to which we compare the data: namely, the ideal-gas model, the semi-ideal model, and the selfconsistent HF model.

\section{EXPERIMENTAL METHODS}

This section reviews the experimental techniques used in this work. After a brief description of our experimental apparatus (Sec. II A), we discuss in Sec. II B condensed fraction and temperature measurements via time-of-flight absorption imaging and point out the limitations of this technique for high condensed fractions. In Sec. II C, we show how they can be overcome using coherent Bragg scattering $[20,21]$.

\section{A. Condensate production}

Our experimental setup employs the standard combination of laser and evaporative cooling to reach Bose-Einstein condensation in a sample of ${ }^{87} \mathrm{Rb}$ atoms, spin polarized in the $\left|F=1 ; m_{F}=-1\right\rangle$ hyperfine ground state. A Zeeman-slowed atomic beam loads a magneto-optical trap in $4 \mathrm{~s}$. After spatial compression (125 ms) and cooling in optical molasses (6 $\mathrm{ms}$ ), the laser-cooled sample is repumped in the $|F=1\rangle$ hyperfine manifold and loaded into an Ioffe-Pritchard-type magnetic trap. The trap, an iron-core electromagnet, is a specific feature of our experiment [22]. The guiding of magnetic flux lines along the ferromagnetic body of the magnet produces strong (when compared to macroscopic, coil-based devices) radial gradients $(1.4 \mathrm{kG} / \mathrm{cm})$, while using a modest current of $30 \mathrm{~A}$. This produces a cigar-shaped, very elongated harmonic potential of the form $V_{\mathrm{ext}}=M \omega_{\perp}^{2}\left(x^{2}+y^{2}\right) / 2$ $+M \omega_{z}^{2} z^{2} / 2$. In this work, differently from [23], radial and axial trapping frequencies are, respectively, $\omega_{\perp} / 2 \pi$ $=413(5) \mathrm{Hz}$ and $\omega_{z} / 2 \pi=8.69(2) \mathrm{Hz}$ at a $10-\mathrm{G}$ bias field.

The combined compression and evaporation sequence typically lasts for $16 \mathrm{~s}$, with the last part of the radio- frequency (rf) evaporation ramp considerably slowed down (to a ramp speed of $200 \mathrm{kHz} / \mathrm{s}$ ) and followed by a 1-s hold time in the presence of an rf shield. This is done in order to allow the system to cross slowly the quantum degeneracy threshold and to relax towards equilibrium. In particular, nonequilibrium shape oscillations that occur in such anisotropic traps upon condensation $[23,24]$ are strongly reduced. To ensure the reproducibility of the temperature in the experiment, the radio frequency $\nu_{0}$ that empties the trap is measured every five experimental cycles, and the rf shield frequency $\nu_{\mathrm{rf}}$ is readjusted in real time to follow slow drifts of the bottom of the trap. In this way, the "trap depth" $\nu_{\mathrm{rf}}-\nu_{0}$ is controlled within $\pm 2 \mathrm{kHz}$. Since we measure $h\left(\nu_{\mathrm{rf}}-\nu_{0}\right) / k_{\mathrm{B}} T$ $\approx 11$ in this final evaporation stage, we estimate the temperature reproducibility to be $\pm 10 \mathrm{nK}$.

\section{B. Time-of-flight imaging}

Information about the atomic cloud is obtained through time-of-flight absorption imaging (see [25] for a detailed account of imaging techniques and [26] for details of the implementation in our experiment). This widely employed method will be referred to as the "standard method" in the following. Here, two important quantities are measured through a fit to absorption images: the condensed fraction and the temperature. To find the condensed fraction, we use the fitting method described in [25], which assumes that the atomic density can be described by a parabolic TF profile describing the condensate plus an ideal Bose-Einstein distribution with zero chemical potential describing the thermal cloud. The condensed number $N_{0}$ is taken to be the number of atoms integrated under the parabolic profile, while the total atom number $N$ is found by integration over the entire profile.

The temperature is measured through an independent fit to a thermal Bose-Einstein distribution, restricted to the wings of the thermal distribution only, in order to minimize the effect of interactions (see [4,5] and Sec. IV). One typically assumes that the high-energy atoms selected by this procedure behave as if the gas were ideal and extracts effective temperatures $T_{x}$ and $T_{z}$ from the cloud sizes-for instance, $k_{\mathrm{B}} T_{x}=M \omega_{\perp}^{2} R_{\mathrm{th}}^{2} /\left[1+\left(\omega_{\perp} t\right)^{2}\right]$, with $R_{\mathrm{th}}$ the radial size. We observe a systematic variation of the temperature $T_{z}$ measured along the long axis with the size of the exclusion region. This effect was more pronounced with increasing condensed fraction and disappeared above $T_{c}$. On the contrary, the radial temperature $T_{x}$ was barely affected by the actual size of the exclusion region, provided it was chosen larger than the condensate radius and sufficiently small to conserve a reasonable signal-to-noise ratio (5\% or less variation). For this reason, we infer the initial temperature from the radial value $T_{x}$ only [28]. This thermometry procedure assumes a nearly ballistic expansion. Collisional effects in time of flight may invalidate this assumption, and it is important to estimate their importance to quantify the accuracy of our measurements. We defer this discussion to Secs. IV and V.

\section{Selective displacement of the condensate using coherent Bragg scattering}

The method described in the last subsection rests on the clean distinction between the condensed and thermal compo- 
nents. However, for cold samples $\left(k_{\mathrm{B}} T \lesssim \mu\right)$, the condensate radius after time of flight is larger than the extension of the thermal cloud. The details of the thermal distribution are therefore "buried" under the condensate density profile, and a reliable fit is not possible. We have found no difficulties for $N_{0} / N \sim 60 \%\left(T / T_{c}^{0} \sim 0.5\right)$, while still being able to extract valuable information for condensed fractions close to $80 \%$ $\left(T \gtrsim 0.3 T_{C 0}\right)$. For higher condensed fractions, the signal to noise on the wings is too low to perform a reliable fit.

In this section, we describe an improvement of the timeof-flight technique that exploits the dramatic difference between the two components in momentum space $[2,20,21]$. Our primary motivation for introducing this method is to improve the resolution at low temperatures, a key advantage in the condensed fraction measurements presented below. The principle of the technique is to transfer the whole condensate to a center-of-mass momentum state with two photon recoil velocities (we define the recoil velocity as $v_{R}$ $=h / M \lambda_{\mathrm{L}} \approx 5.8 \mathrm{~mm} / \mathrm{s}$, where $\lambda_{\mathrm{L}}=780 \mathrm{~nm}$ is the Bragg laser wavelength), while leaving the thermal atoms essentially unaffected. As shown in Fig. 1, after a subsequent time of flight (typically $20 \mathrm{~ms}$ ), the condensate has moved away from the center of the thermal cloud, allowing us to perform separate fits and revealing previously hidden information about the thermal component. This technique is related to the filtering scheme used in [29] to study collective modes in the presence of a weak optical lattice at finite temperatures.

Momentum transfer is achieved by exposing the sample to two counterpropagating laser beams detuned with respect to each other. The moving lattice formed this way can Braggdiffract the atoms, promoting them to a two-recoil momentum state. In principle, Bragg scattering is sensitive to the atomic momentum distribution [20,21]. However, if the spectral width of the Bragg pulse, Fourier limited by the pulse duration $T$ to $\Delta \nu_{\mathrm{F}} \sim 1 / T$, is much larger than the condensate Doppler width $\Delta \nu_{0} \sim 2 \nu_{\mathrm{R}} / L$, the momentum sensitivity of Bragg diffraction is lost and almost complete transfer of the condensate ( $\pi$ pulse) is achieved. On the other hand, if the thermal component spectral width $\Delta \nu_{\text {th }}$ $\sim 2 \nu_{\mathrm{R}} / \lambda_{\mathrm{T}}\left(\lambda_{\mathrm{T}}=\sqrt{2 \pi \hbar^{2} / m k_{\mathrm{B}} T}\right.$ is the thermal de Broglie wavelength) is much larger than $\Delta \nu_{\mathrm{F}}$, Bragg diffraction is then a momentum-selective process, and most of the thermal atoms, being off resonant with the Bragg beams, stay at rest in the laboratory frame. This filtering technique thus makes possible independent manipulation of the condensed and noncondensed atoms.

The experimental setup that we use to generate the Bragg beams is identical to the one used in [23]. The $\pi$ Bragg pulse is applied after a sudden switch-off of the trapping potential and a 2-ms time of flight. This decreases the condensate and thermal cloud densities by a factor of order $\left(\omega_{\perp} t\right)^{2} \sim 30$, making any further mutual interactions negligible. This avoids in particular $s$-wave collisions between atoms with different momenta [30] and suppresses the equilibration of the thermal cloud with the displaced condensate. The Bragg beams are counterpropagating and parallel to the elongated axis of the trap. Since the condensate mean-field energy is then almost entirely released in the transverse directions, perpendicular to the diffraction axis, the ratio between the condensate and thermal cloud spectral widths is

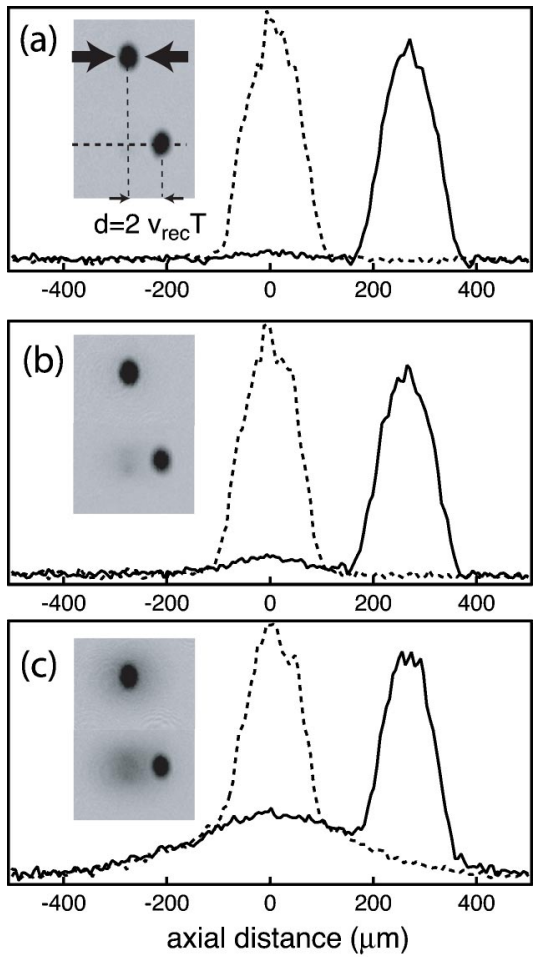

FIG. 1. Bragg diffraction as a condensate filter. (a), (b), and (c) correspond to $92 \%, 85 \%$, and $30 \%$ condensed fraction, respectively $\left(T / T_{\mathrm{c}}^{0} \approx 0.2,0.35\right.$, and 0.8$)$. Two-dimensional absorption images are shown, with a cut along the direction of the trap weakest axis ("axial cut"). The top images show regular absorption images for a 24.27-ms time of flight (dashed lines in the cut). The bottom images, also shown as the solid line in the axial cut, corresponding to the same temperatures and atom numbers within experimental reproducibility, have been taken after applying a moving optical lattice tuned to realize a $\pi$ Bragg pulse, transferring two photon recoils to almost all condensed atoms (velocity $1.1 \mathrm{~cm} / \mathrm{s}$ ). The distance traveled by the condensate is verified to be $250 \mu \mathrm{m}$, corresponding to a free flight of $22.27 \mathrm{~ms}$ after the Bragg pulse. As can be seen, the thermal cloud is barely affected, due to its much larger extent in momentum space.

$$
\left(\frac{\Delta \nu_{0}}{\Delta \nu_{\text {th }}}\right)_{\text {T OF }} \sim\left(\frac{\omega_{z}}{\omega_{\perp}}\right) \sqrt{\frac{\mu}{k_{\mathrm{B}} T}}
$$

and remains smaller than 1 even at very low temperatures. Note that this would not have been the case for Bragg beams parallel to a radial axis, where the momentum width is larger by a factor $\left(\omega_{\perp} / \omega_{z}\right)$ due to the released mean-field energy.

To find the condensate fraction, we simply count the number of diffracted atoms and identify it with the condensed number. To obtain a meaningful measurement of the condensed fraction, two conditions have to be met. First, almost all condensate atoms should be diffracted. Transfer efficiencies as high as $97 \%$ were observed for the coldest clouds we have produced, indicating that only a few percent of the condensate population remains at rest; this is comparable to the $\pm 2 \%$ root-mean-square fluctuations of the diffraction efficiency that we have measured experimentally. Second, the diffracted number of thermal atoms should be small to obtain 

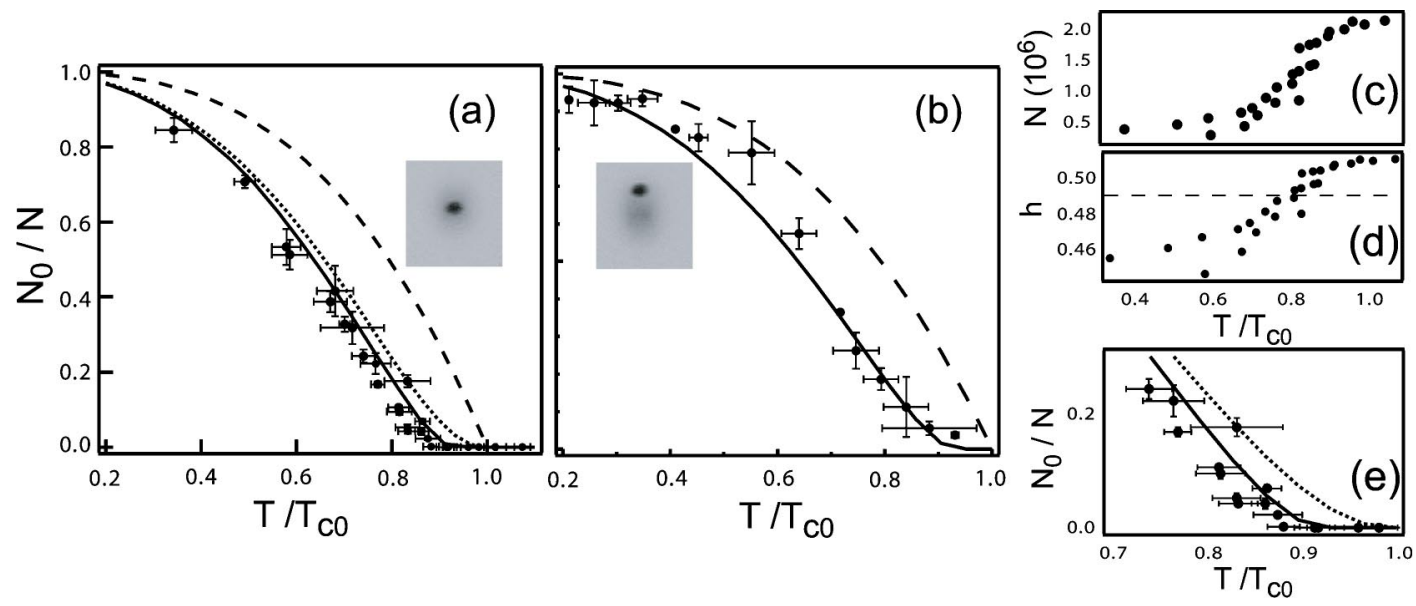

FIG. 2. Condensed fraction as a function of reduced temperature. (a),(b) Experimental data after averaging are shown as solid circles, with statistical error bars. Data in (a) were taken using the standard time-of-flight technique, while data in (b) were measured with the Bragg filtering scheme and extend to lower temperature, down to $T \approx 0.2 T_{\mathrm{C} 0}$, whereas the standard method is limited to $T \approx 0.4 T_{\mathrm{C} 0}$. Lines show theoretical expectations according to an ideal gas calculation, including finite-size effects (dashed line), a "semi-ideal" model that neglects interactions within the thermal cloud (dotted line), and a self-consistent HF calculation (solid line). Due to a different average atom number, the parameter $\bar{\eta}$ that controls the importance of interactions is different in each case, $\bar{\eta}=0.49$ and $\bar{\eta}=0.47$ for (a) and (b), respectively. The variation in total number due to evaporative cooling across the data set in (a) is shown in (c), and the corresponding variations of $\eta$ in (d) (note the vertical scale, extending over no more than $5 \%$ of the average value). The average $\bar{\eta}=0.49$ is shown by the dashed line. In (e), we show an enlargement of (a) around the critical temperature, to highlight the importance of making the full HF calculation to reproduce the trend seen in the data.

a sensible measurement of the condensed number. We estimate the diffracted fraction of thermal atoms $f_{\text {th }}$ in the impulse approximation discussed in [31]. In this approximation, well justified for the low densities considered here, the number of atoms diffracted from the thermal cloud is taken to be proportional to the thermal part of the momentum distribution, integrated over the directions perpendicular to the axis of the Bragg beams. Treating the thermal cloud as an ideal Bose gas in a first approximation, we find for a resonant $\pi$ pulse,

$$
f_{\text {th }}=\frac{\pi^{2}}{4 \Delta \nu_{\text {th }} T} \frac{\left[g_{5 / 2}(1)-g_{5 / 2}\left\{\exp \left[-32\left(\nu_{\mathrm{R}} / \Delta \nu_{\mathrm{th}}\right)^{2}\right]\right\}\right]}{g_{3 / 2}(1)},
$$

where $\Delta \nu_{\mathrm{th}}=2 v_{\mathrm{R}} / \sqrt{2 \pi} \lambda_{\mathrm{T}}$. Here and below, the Bose functions are defined by $g_{\propto}(u)=\Sigma_{j \geqslant 1} u^{j} / j^{\propto}$. The second term in brackets arises from off-resonant excitations, which are negligible for the condensate but sizable for the thermal component. The quantity $f_{\text {th }}$ varies from $6 \%$ at $400 \mathrm{nK}\left(T \sim 0.8 T_{\mathrm{c}}^{0}\right.$ or $\left.N_{0} / N \sim 20 \%\right)$ to $20 \%$ at $150 \mathrm{nK}\left(T \sim 0.2 T_{\mathrm{c}}^{0}\right.$ or $\left.N_{0} / N \sim 90 \%\right)$. The ratio between the number of thermal atoms to the number of BEC atoms in the diffracted peak is given by (1 $\left.-f_{\mathrm{c}}\right) / f_{\mathrm{c}} f_{\mathrm{th}}$, where $f_{\mathrm{c}}=N_{0} N$ is the condensed fraction, and it should remain small for the technique to work. All in all, we estimate from this simplified calculation that the Bragg filter is a useful technique in a window $5 \%<N_{0} / N<95 \%$, the upper bound being set by the first condition (complete condensate transfer) and the lower by the second (the number of condensed atoms larger than the number of thermal atoms in the diffracted peak). Outside of this window, it becomes suspect to identify unambiguously the diffracted order with the condensate. Despite this, the technique represents a signifi- cant improvement over the standard method, as we will see in the next sections.

\section{CONDENSED FRACTION OF THE INTERACTING GAS VERSUS TEMPERATURE}

With the tools of Sec. II in hand, we can investigate the behavior of the condensed fraction as a function of temperature. This quantity is of primary importance: the possibility to measure it directly in trapped gases is in stark contrast with the situation in traditional, bulk superfluids where such measurements are intrinsically difficult [3]. We have done this measurement in two steps, first by using the standard method and second by taking advantage of the enhanced resolution of the Bragg filtering scheme.

\section{A. Standard time-of flight measurements}

Using the standard analysis technique (i.e. no Bragg filtering), we investigate in this section a temperature interval ranging from $T \approx 1.1 T_{\mathrm{c}}^{0}$ down to $T \approx 0.3 T_{\mathrm{c}}^{0}$, with approximately $2 \times 10^{6}$ atoms at the transition. Figure 2 shows the measured condensed fraction as a function of $T / T_{\mathrm{c}}^{0}$. Each point results from an averaging over several (typically five) realizations under identical conditions. The expectation for an ideal gas, including finite-size effects [2], lies distinctly above our experimental data. The difference can be attributed to interactions, as shown by the far better agreement with the self-consistent HF calculation (solid line). The observed reduction of the condensed fraction contrasts with the homogeneous case [32], where the condensed and normal components overlap everywhere and where it is energetically favorable to increase the condensed fraction to diminish the 
exchange interaction energy among excited states.

An interesting property, first pointed out in [33], is the scaling behavior of all thermodynamical quantities, which depend only on the reduced temperature $T / T_{\mathrm{c}}^{0}$ and on the parameter $\eta$ which controls the magnitude of two-body repulsion [34]:

$$
\eta=\frac{\mu_{\mathrm{TF}}\left[N_{0}=N\right]}{k_{\mathrm{B}} T_{\mathrm{c}}^{0}} \approx 1.57\left(\frac{a}{\bar{\sigma}}\right)^{2 / 5} N^{1 / 15} .
$$

Alternatively, one can express $\eta$ as the ratio between the two characteristic lengths in the uniform problem-the scattering length $a$ and the de Broglie wavelength $\lambda_{0}$ for $T=T_{\mathrm{c}}^{0}-$ as $\eta \approx 1.07\left(a / \lambda_{0}\right)^{2 / 5}$. The power $2 / 5$ reflects the presence of the trapping potential. A typical value in our experiment is $\eta$ $=0.5$, while previous experiments (for instance, [4]) correspond to $\eta \sim 0.3-0.4$. Thus, the effect of two-body interactions is stronger in the work reported here, which explains to some extent the clarity with which we observe deviations from ideal-gas behavior.

In the experiment, because the total number $N$ drops with $T$ due to evaporation and losses, $\eta$ decreases slightly across the temperature range, from 0.51 above $T_{\mathrm{c}}$ to 0.47 at low temperature [see Figs. 2(c) and 2(d)]. To compare with theory, we use the average number of atoms across the data set, $\vec{N} \approx 1.2 \times 10^{6}$, and the corresponding $\bar{\eta}=0.49$. This does not lead to a discernible variation of the HF prediction at the scale of the graph, because of the weak $N^{1 / 15}$ dependence of $\eta$. This behavior emphasizes the scaling behavior exhibited by trapped Bose gases with large atom numbers [33].

Finally, we note that the data in the vicinity of $T_{\mathrm{c}}$, shown in Fig. 2(e), emphasize the necessity of the full HF treatment of the thermal component to understand quantitatively the thermodynamic properties. As a matter of fact, the semi-ideal model (dashed line) predicts a condensed fraction systematically higher than the one we observe. This is clear evidence for mutual interaction between the condensed and noncondensed components. We will return later to this point, which is an important conclusion to be drawn from this work.

\section{B. Enhanced resolution of low thermal fraction}

As discussed earlier, at low temperatures $T \lesssim 0.4 T_{C}$, the standard procedure is unable to extract faithfully the properties of the thermal cloud. Thanks to the Bragg filtering technique introduced in Sec. II C, this difficulty can be overcome and a very small thermal fraction can be detected. The condensed fraction measured this way is plotted in Fig. 2(b), along with the $\bar{\eta}=0.47$ curve that corresponds the value $\bar{N}$ $\approx 8 \times 10^{5}$ for this set of measurements. Again, we find good agreement with the prediction of the HF model within our uncertainty, even at very low temperatures. One could wonder whether the contribution from collective excitations (quantum and low-energy thermal depletion) could be measured by this technique (the analog of the phonon regime in superfluid ${ }^{4} \mathrm{He}$ ). Unfortunately, according to the estimations of [18], they are always small when compared to the contribution of single-particle excitations in the temperature range we explore and compared to the estimated sensitivity of the Bragg technique (Sec. II C). Only by increasing the dilute- ness parameter $\sqrt{n_{0}(0) a^{3}}$ significantly could this regime become observable experimentally with the techniques described here.

\section{MEASUREMENT OF THE OVERALL AND THERMAL EXPANSION ENERGIES}

Another quantity that can be measured from time-of-flight expansion is the release energy $[2,4,5]$, the sum of the kinetic and interaction energy released at the trap cutoff and available for the expansion of the whole cloud. In an anisotropic trap such as ours $\left(\omega_{\perp} / \omega_{z} \approx 51\right)$, almost all the interaction energy converts into radial expansion velocity. The radial expansion of the cloud for $t \gg \omega_{\perp}^{-1}$, observed in the $y$ direction, proceeds at an overall speed $v_{y}$, fixed by the expansion energy

$$
E_{y}=\frac{1}{2} M v_{y}^{2}=\frac{1}{3} E_{\mathrm{kin}}+\frac{1}{2} E_{\mathrm{int}},
$$

which, if scaled by the characteristic $N k_{\mathrm{B}} T_{\mathrm{c}}^{0}$, is a universal function of $\eta$ and $T / T_{\mathrm{c}}^{0}$. We discuss in the Appendix I how to calculate the kinetic and interaction energies in the HF approximation.

Experimentally, one measures $E_{y}$ directly from the rootmean-square cloud radius, according to $\left\langle y^{2}\right\rangle=v_{y} t$ [4,5], without resorting to a detailed fitting model. This expression assumes negligible relaxation between the axial and radial degrees of freedom. We have plotted the measured value of $E_{y}$ in Fig. 3(a) for same data as those shown in Fig. 2(b), where the Bragg filter has been used $(\eta \approx 0.47$ for these data). As expected, the kinetic energy of the thermal cloud dominates close to $T_{\mathrm{c}}$, with a small contribution of the meanfield energy, whereas the interaction energy of the condensate $E_{0}$ is the most important term at low temperature. The $\mathrm{HF}$ curve connects these two limiting cases and reproduces well our observations.

A key advantage in our situation is the ability to analyze separately the condensate and thermal cloud thanks to the Bragg filtering scheme and, therefore, to measure the release energy of the thermal cloud alone. To avoid the condensate, we estimate the thermal cloud rms radius from a fit to a radial cut to the image [see inset in Fig. 3(b)]. The release energy of the thermal cloud measured this way is shown in Fig. 3(b), together with the calculated value:

$$
E_{y}^{\text {(th) }}=\frac{1}{3} E_{\mathrm{kin}}+\frac{k_{\mathrm{B}} T}{2}\left(\zeta_{0}+\zeta_{\mathrm{th}}\right) .
$$

The quantities $\zeta_{0}$ and $\zeta_{\text {th }}$ represent the scaled mean-field energy corresponding to the repulsion felt by a thermally excited atom due the condensate and to the remaining thermal atoms, respectively. They are defined more precisely in the Appendix I. Although the difference with the noninteracting curve is less pronounced at very low temperatures, where the thermal energy is very low, close to $T_{\mathrm{c}}$, these results emphasize the important role of interactions and the good agreement with HF theory once again. 

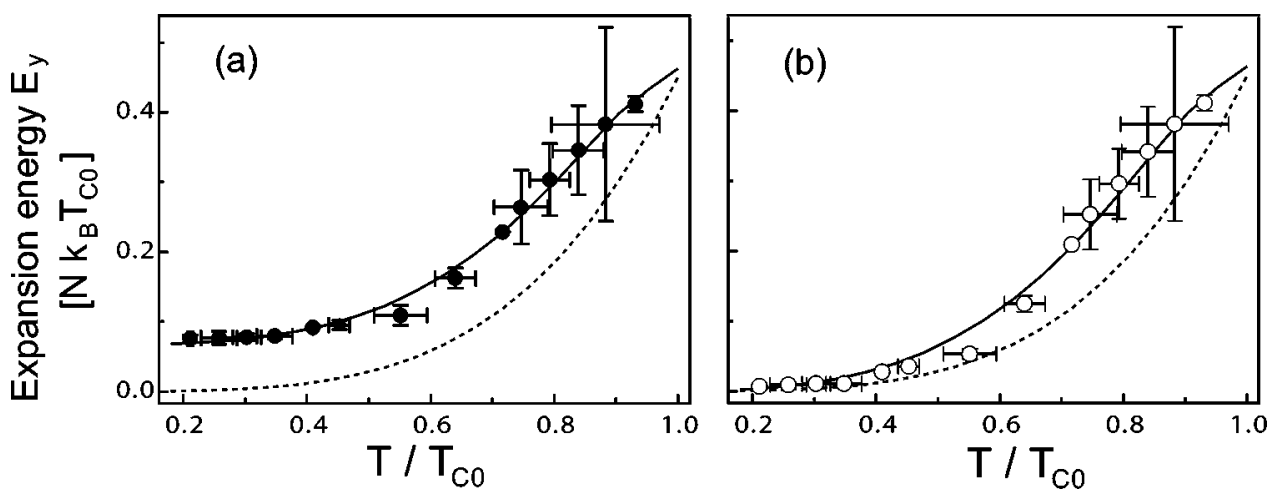

FIG. 3. Expansion energy in the radial $x$ direction as a function of temperature. Solid circles in (a) correspond to the whole cloud and open circles in (b) to the thermal component only. The data are taken from the same set as in Fig. 2(b). The solid lines on the graphs show the same quantities predicted by the self-consistent HF model, with $\eta=0.47$. The dotted line is the expansion energy (kinetic only) of an ideal cloud.

\section{FURTHER EVIDENCE FOR MUTUAL INTERACTION BETWEEN THE CONDENSED AND THERMAL COMPONENTS}

From images taken employing the Bragg filtering scheme, it is also possible to examine a radial cut to the profile of the thermal cloud, as shown in Fig. 4(a). As the initial kinetic energy of the thermal cloud is typically much larger than its mean-field energy, one expects that the density distribution after time of flight reflects at least approximately the initial
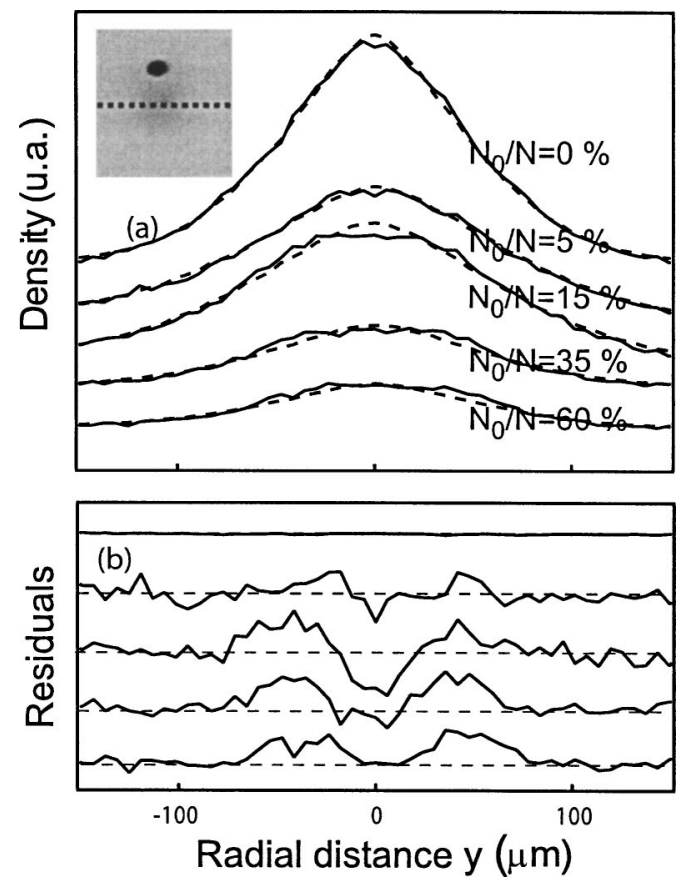

FIG. 4. Radial density profile of the thermal cloud. The dashed line in the inset shows the axis along which the profiles are taken. (a) Radial cut of the column density profile of the thermal cloud after $24.3 \mathrm{~ms}$ time of flight (solid line) and the best fit to an ideal Bose-Einstein distribution (dashed line). The Bragg filter has been employed to separate the thermal and condensed components. The condensed fraction is indicated in each case. (b) Residual of the fit for each case in (a). momentum distribution, which does not display the "hole" present in the density distribution in the trap (see the Appendix I for further discussion). This is indeed the case: the measured profiles show a monotonic behavior near the center of the cloud [35]. Nevertheless, the density distribution we observe is somewhat flatter in the central region than the ideal-gas distribution used in the analysis, as shown by the residuals of a fit to the radial profile [Fig. 4(b)]. Above $T_{\mathrm{c}}$, the effect disappears, which indicates that a flatter profile is not simply an artifact of our measurement method. Furthermore, such a behavior is to be expected if the condensate mean field repels the thermal cloud in the early stage of the expansion, since interactions tend in general to make the density profile more uniform.

\section{A. Evidence for nonballistic expansion}

As stated in Sec. II, this repulsion effect is not taken into account in our fitting procedure, which assumes an ideal Bose distribution to fit the profile and ballistic expansion to deduce temperature from the cloud sizes. To investigate further the validity of the analysis, we begin by plotting in Figs. 5(a) and 5(b) the aspect ratio of both components, as a function of the reduced temperature. One sees from these graphs that the simple model of a TF condensate on top of an ideal thermal background is not sufficient to account for the data. Indeed, for our trapping frequencies and for the time of flight $t=22.3 \mathrm{~ms}$ used in these measurements, one would expect from this model an aspect ratio of 1.17 for the condensate and 0.77 for the thermal cloud. Both deviate from these values and vary with temperature, indicating that the expansion dynamics is more complex than assumed by the analysis model. Note that although this model fails to describe fully the expansion dynamics, the observed deviation from ballistic expansion remains small.

As already pointed out, the obvious weakness of the analysis model is the neglect of collisional effects. We recall that the expansion model is basically motivated by the absence of a more elaborate theory to which we could compare our observations. It is, however, of interest to quantify the error level on temperature measurements, which we will do 

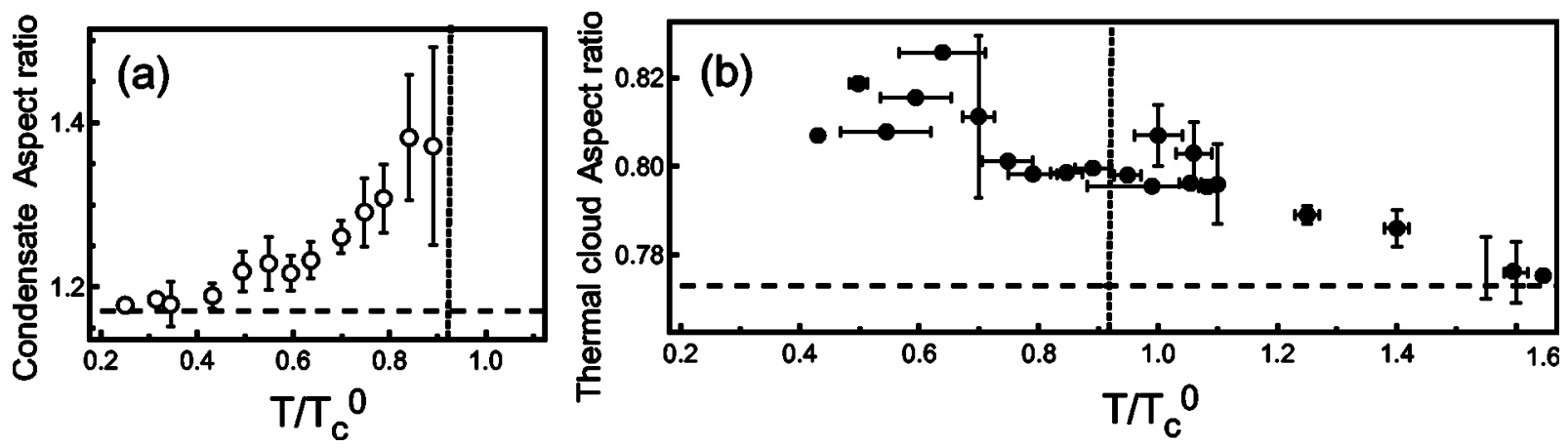

FIG. 5. Deviation from ballistic expansion. The aspect ratios of the condensed (a) and thermal (b) components of mixed clouds, after 22.3 ms of free expansion, are plotted as a function of the reduced temperature. The aspect ratios for noncondensed clouds, analyzed in more detail in [27], are also shown for comparison. The horizontal dashed lines indicate the aspect ratio of a TF condensate and an ideal thermal gas, respectively, and the vertical dotted lines show the critical temperature including mean-field and finite-size effects.

in the remainder of this section at the gross estimate level.

Interaction-driven forces that affect the expansion can be divided into two distinct classes [36]: hydrodynamic forces on the one hand, predominant above $T_{\mathrm{c}}$ in [27], and meanfield repulsion on the other, which play a minor role above $T_{\mathrm{c}}$, but become increasingly important with decreasing $T$ due to the presence of the condensate [37]. Both effects are roughly speaking comparable in magnitude, and from our observations above $T_{c}$ and HF calculations (see the I), we estimate an upper bound on the systematic error on temperature measurements, from $10 \%$ close to $T_{\mathrm{c}}$ (mostly due to hydrodynamic behavior) to $20 \%$ well below (mostly due to repulsion by the condensate), compatible with the observed deviation from the ideal gas expansion.

This is an upper bound because the temperature is found through a fit to the wings of the distribution the only, which is expected to reduce effect of mean-field repulsion. The close agreement we find between the calculated release energy and the measured one in Fig. 3(b), where we recall that the radial size used to deduce the expansion energy was found through a fit to the full profile, seems to indicate that fitting to the wings avoids counting most of the repulsion energy in the determination of the temperature, as expected. However, this need not be true for the hydrodynamic relaxation, which affects directly the momentum distribution. Just as above $T_{\mathrm{C}}$, hydrodynamic forces are thus likely to be the dominant source of systematic error in most of the temperature range in elongated traps such as ours.

\section{B. Compression of the condensate by the thermal cloud}

We conclude this section by examining the axial length of the condensate, which is reasonably immune to the effects discussed above because axial expansion of the condensate is very slow: the measured length thus stays close to the in-trap length. From condensed fraction measurements, it is clear that the semi-ideal model is not sufficient to reproduce our results, meaning that the mutual interaction between condensed and noncondensed atoms is observable. This is also seen from the length of the condensate inferred from the two-component fit, which is reduced when compared to the TF length calculated with the number of condensate atoms we measure. A quantitative comparison can be made only by taking into account the slow axial expansion. In the absence of a complete theory, we assume that the axial length is rescaled from the equilibrium length by the same factor as a condensate in the TF regime, $b_{z} \approx 1+\pi \omega_{z}^{2} t / 2 \omega_{\perp} \approx 1.04$ for our parameters $[39,40]$. With this assumption, we find our data to be in reasonable agreement with the HF length, whereas the TF prediction is found systematically too high (see Fig. 6). This reduction can be attributed to the thermal cloud compression discussed in the I: at equilibrium, the shell of thermal atoms surrounding the condensate exerts a force towards the trap center, reducing its extension when compared to a "free" TF condensate.

\section{CONCLUSION}

In this paper, we have investigated experimentally the thermodynamics of a trapped, interacting Bose gas over a wide range of temperatures, from $T_{\mathrm{c}}$ down to $0.2 T_{\mathrm{c}}$. We have used the standard time-of-flight analysis, complemented by the use of coherent Bragg scattering, to filter the condensate out of the thermal cloud. The latter technique allows us to reach lower temperatures and higher condensed fractions than those accessible by the usual method. We have investigated primarily two quantities: the condensed fraction and

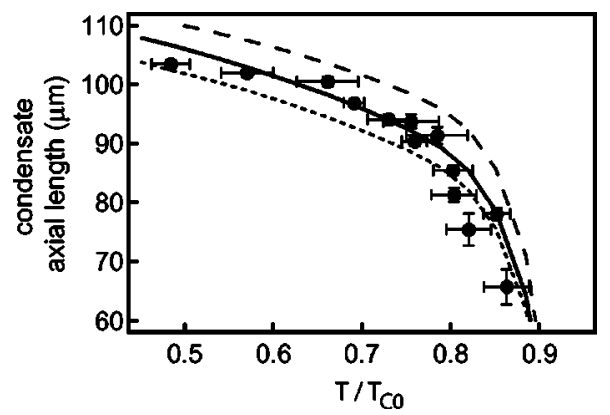

FIG. 6. Compression of the condensate by the thermal cloud. The axial length of the condensate as measured in the absorption images is shown, with the TF (dashed line) and the HF predictions for the trapped condensate (dotted line) and after rescaling by the same factor as a TF condensate (solid line). 
the (radial) release energy. The data display without ambiguity an interacting gas behavior and are in agreement at a few percent level with a Hartree-Fock description of the mixed cloud. This highlights the pertinence of this simple description of the interacting, trapped Bose gas at finite temperature. Moreover, this gives evidence for a mutual interaction between the condensate and the thermal cloud close to $T_{\mathrm{c}}$. Although these effects are small, they are measurable and should be taken into account in precise comparisons to the theory of finite-temperature, Bose-Einstein condensed gases.

A more thorough quantitative test of theory for groundstate occupation and similar thermodynamic properties is, however, hindered by the lack of theory to understand the expansion of a mixed cloud. For instance, the data in Fig. 2(a) lie systematically slightly below the theory, which may be due to an underestimation of the condensed fraction or the temperature: as described above, we use an ideal Bose distribution to fit the thermal component. This assumption contradicts the principal conclusion of this paper, that ideal-gas approximations are far from sufficient at the desired level of accuracy. Although the experiments described in this paper suggest an accuracy on temperature of order $10 \%$ at least, we have also shown that the density profile deviates from an ideal gas near the center of the cloud and stressed that hydrodynamic and mean-field effects in the expansion are not properly accounted for. Systematic errors may thus still be present, and a better accuracy is not guaranteed with the methods used in this paper. Numerical work-for instance, along the lines of [41] — may help study the expansion of mixed clouds and improve the standard analysis procedure.

\section{ACKNOWLEDGMENTS}

We would like to thank D. Guéry-Odelin, S. Giorgini, J. Dalibard, G. V. Shlyapnikov, J. Retter, and D. Boiron for useful discussions and comments on the manuscript, as well as D. Clément for his work on a related project. J.H.T. acknowledges support from the Châteaubriand Program, CNRS, and NSERC, and M.H. from IXSEA. This work was supported by Délégation Générale de l'Armement, the European Union (Cold Quantum Gas network), INTAS (Contract No. 211-855), and XCORE.

\section{APPENDIX: SIMPLE MODELS OF A TRAPPED BOSE-EINSTEIN CONDENSED GAS AT FINITE TEMPERATURES}

In this appendix, we briefly summarize the three different models to which we compare our observations. We use as a temperature scale the critical temperature of an ideal gas in the thermodynamic limit [2], $k_{\mathrm{B}} T_{\mathrm{c}}^{0}=\hbar \bar{\omega}[N / \zeta(3)]^{1 / 3}$, where $\zeta$ is the Riemann function and $\bar{\omega}=\omega_{\perp}^{2 / 3} \omega_{z}^{1 / 3}$ is the geometrical mean of the trapping frequencies. We make three key assumptions [labeled (i) - (iii) in the following] to simplify the theoretical description. For the condensate, we suppose that (i) the condition $N_{0} a / \bar{\sigma} \gg 1$ holds, where $\bar{\sigma}=\sqrt{\hbar / M \bar{\omega}}$ is the mean ground-state width and $a$ the scattering length. This ensures that the condensate is in the TF regime. At very low temperatures, where the condensed fraction is almost unity, this gives the condensate density $n_{0}=n_{\mathrm{TF}}$ as

$$
n_{\mathrm{TF}}(\mathbf{r})=\left[\mu_{\mathrm{TF}}-V_{\mathrm{ext}}(\mathbf{r})\right] / U,
$$

where $U=4 \pi \hbar^{2} a / M$ is the mean-field coupling constant. The $\mathrm{TF}$ chemical potential is $\mu_{\mathrm{TF}}=(\hbar \bar{\omega} / 2)\left(15 N_{0} a / \bar{\sigma}\right)^{2 / 5}$. For the thermal cloud, we assume (ii) $k_{\mathrm{B}} T \gg \hbar \omega_{\perp}$ for the semiclassical approximation to hold and (iii) use a mean-field description which treats thermal atoms as independent particles evolving in a self-consistent static potential $V_{\text {eff }}(\mathbf{r}$ ) (see below). The distribution function in phase space for thermal particles then reads

$$
f(\mathbf{r}, \mathbf{p})=\frac{1}{e^{\beta[H(\mathbf{r}, \mathbf{p})-\mu]}-1},
$$

with the semiclassical Hamiltonian $\mathcal{H}(\mathbf{r}, \mathbf{p})=\mathbf{p}^{2} / 2 M$ $+V_{\text {eff }}(\mathbf{r})$, the chemical potential $\mu$, and $\beta=1 / k_{\mathrm{B}} T$. The particle density distribution for the thermal component $n_{\text {th }}$ is found by integration over momenta and reads

$$
n_{\mathrm{th}}(\mathbf{r})=\frac{1}{\lambda_{\mathrm{T}}^{3}} \quad g_{3 / 2}\left\{\exp \left[\beta\left(\mu-V_{\mathrm{eff}}(\mathbf{r})\right)\right]\right\} .
$$

Each model detailed below is thus specified by the precise form of $V_{\text {eff }}$ : in the ideal-gas model, $V_{\text {eff }}$ reduces to the trapping potential, in the semi-ideal model, it includes the mean field of the condensate only, and in the HF model, it also takes the mean field of the thermal atoms into account.

\section{Ideal-gas model}

The simplest approximation neglects all interactive contributions to the effective potential, which reduces to the trapping field:

$$
V_{\text {eff }}-\mu=V_{\text {ext }}-\mu .
$$

The thermodynamic quantities follow the ideal-gas laws (see, for instance, [2]) and impose in particular $\mu=0$ below $T_{\mathrm{c}}$ and $N_{0} / N=1-\left(T / T_{\mathrm{c}}^{0}\right)^{3}$ for $T \leqslant T_{\mathrm{c}}^{0}$. This ideal-gas description is thus incompatible with the existence of a TF condensate, which implies $\mu=\mu_{\mathrm{TF}}>0$, and it should be considered as a reasonable approximation only for $k_{\mathrm{B}} T \gg \mu$.

\section{Semi-ideal model}

The repulsion of the thermal cloud by condensed atoms is taken into account in the so-called "semi-ideal" model $[10,11]$, which considers a TF condensate containing $N_{0}<N$ atoms plus a quantum-saturated, ideal thermal gas moving in the combined trapping plus condensate mean-field potential:

$$
V_{\mathrm{eff}}(\mathbf{r})-\mu=V_{\mathrm{ext}}(\mathbf{r})+2 U n_{\mathrm{TF}}(\mathbf{r})-\mu_{\mathrm{TF}} .
$$

The factor of 2 accounts for exchange collisions between atoms in different quantum states [2]. Note that the condensed atom number $N_{0}$ should be found self-consistently for a given $T$ under the constraint that the total atom number be fixed. 
The semi-ideal model correctly predicts the most important feature in the static density profile of trapped interacting Bose gases. As soon as the condensed fraction is larger than a few percent, the density of the condensate greatly exceeds the density of the thermal component. Therefore, the condensate mean field is stronger and repels the thermal cloud from the center of the trap, digging a hole in the thermal density distribution.

\section{Self-consistent Hartree-Fock model}

Although qualitatively correct and appealing because of its simplicity, the semi-ideal model is not sufficient to describe precisely our experiments. Interactions among thermal atoms and the backaction of the thermal cloud on the condensate have to be taken into account, which we do here in the HF approximation [12-18], corresponding to a selfconsistent potential:

$$
V_{\mathrm{eff}}(\mathbf{r})-\mu=V_{\mathrm{ext}}(\mathbf{r})+2 U n_{0}(\mathbf{r})+2 U n_{\mathrm{th}}(\mathbf{r})-\mu .
$$

The equilibrium condensate density is no longer simply given by the TF profile, but depends also on the thermal density through

$$
n_{0}(\mathbf{r})=\frac{\mu-V_{\mathrm{ext}}(\mathbf{r})-2 U n_{\mathrm{th}}(\mathbf{r})}{U} .
$$

A self-consistent numerical solution with a fixed atom number $N$ fixes the finite- $T$ chemical potential, $\mu=U n_{0}(0)$ $+2 U n_{\mathrm{th}}(0)$, from which all other quantities can be determined. For instance, the interaction energy, which contains mixed terms describing the mutual influence of the condensate and the thermal cloud, can be written as $E_{\text {int }}=E_{0}$ $+k_{B} T\left(2 \zeta_{0}+\zeta_{\mathrm{th}}\right)$, where

$$
E_{0}=\frac{U}{2} \int d^{(3)} \mathbf{r} n_{0}(\mathbf{r})^{2},
$$

$$
\begin{gathered}
\zeta_{0}=\frac{U}{k_{\mathrm{B}} T} \int d^{(3)} \mathbf{r} n_{0}(\mathbf{r}) n_{\mathrm{th}}(\mathbf{r}), \\
\zeta_{\mathrm{th}}=\frac{U}{k_{\mathrm{B}} T} \int d^{(3)} \mathbf{r} n_{\mathrm{th}}(\mathbf{r})^{2} .
\end{gathered}
$$

The kinetic energy is entirely due to the thermal cloud in the $\mathrm{TF}$ approximation and reads

$$
E_{\mathrm{kin}}=\int \frac{1}{h^{3}} d^{(3)} \mathbf{r} d^{(3)} \mathbf{p} \frac{\mathbf{p}^{2}}{2 M} f(\mathbf{r}, \mathbf{p}) .
$$

Numerical solution of the HF model displays two additional features compared to the semi-ideal model. First, mean-field interactions lower the critical temperature for Bose-Einstein condensation [27-32]. Second, there is a backaction of the thermal cloud on the condensate: the mean field exerted by the shell of thermal atoms surrounding the condensate acts in return to compress it, increasing its density and reducing its axial length $L_{0}$ according to

$$
L_{0}^{2}=\frac{2 g}{m \omega_{z}^{2}}\left\{n_{0}(0)+\frac{2}{\lambda_{\mathrm{T}}^{3}}\left\{g_{3 / 2}\left(e^{-g n_{0}(0) / k_{\mathrm{B}} T}\right)-g_{3 / 2}[1]\right\}\right\} .
$$

This compression effect is directly observed in Sec. V B and indirectly through the measurements of condensed fraction in Sec. III.

A further step forward would be to include collective effects in the model. However, Ref. [42] points out that lowenergy, collective excitations cause a minute change in the thermodynamic properties of the system even at relatively low temperatures $T \lesssim \mu$. Another approach, based on quantum Monte Carlo calculations [43,44], has confirmed that the HF approximation could reproduce the thermodynamics of the trapped clouds to a very good accuracy.
[1] For a recent review, see Nature Insight 416 (2002).

[2] F. Dalfovo, S. Giorgini, L. P. Pitaevskii, and S. Stringari, Rev. Mod. Phys. 71, 463 (1999).

[3] P. E. Sokol, in Bose-Einstein Condensation, edited by A. Griffin, D. W. Snoke, and S. Stringari (Cambridge University Press, Cambridge, England, 1993), p. 51.

[4] J. R. Ensher, D. S. Jin, M. R. Matthews, C. E. Wieman, and E. A. Cornell, Phys. Rev. Lett. 77, 4984 (1996).

[5] M.-O. Mewes, M. R. Andrews, N. J. van Druten, D. M. Kurn, D. S. Durfee, and W. Ketterle, Phys. Rev. Lett. 77, 416 (1996).

[6] O. Maragò, G. Hechenblaikner, E. Hodby, and C. Foot, Phys. Rev. Lett. 86, 3938 (2001).

[7] D. J. Han, R. H. Wynar, P. Courteille, and D. J. Heinzen, Phys. Rev. A 57, R4114 (1998).

[8] B. P. Anderson and M. A. Kasevich, Phys. Rev. A 59, R938 (1999).

[9] F. Schreck, L. Khaykovich, K. L. Corwin, G. Ferrari, T. Bourdel, J. Cubizolles, and C. Salomon, Phys. Rev. Lett. 87, 080403 (2001).
[10] A. Minguzzi, S. Conti, and M. P. Tosi, J. Phys.: Condens. Matter 9, L33 (1997).

[11] M. Naraschewski and D. M. Stamper-Kurn, Phys. Rev. A 58, 2423 (1998).

[12] V. V. Goldman, I. F. Silvera, and A. J. Leggett, Phys. Rev. B 24, 2870 (1981).

[13] D. A. Huse and E. Siggia, J. Low Temp. Phys. 46, 137 (1982).

[14] J. Oliva, Phys. Rev. B 39, 4197 (1989).

[15] V. Bagnato, D. E. Pritchard, and D. Kleppner, Phys. Rev. A 35, 4354 (1988).

[16] H. Shi and W.-M. Zheng, Phys. Rev. A 56, 1046 (1997).

[17] H. Shi and W.-M. Zheng, Phys. Rev. A 56, 2984 (1997).

[18] S. Giorgini, L. P. Pitaevskii, and S. Stringari, J. Low Temp. Phys. 109, 309 (1997).

[19] B. D. Busch, C. Liu, Z. Dutton, C. H. Behroozi, and L. V. Hau, Europhys. Lett. 51, 485 (2000).

[20] M. Kozuma, L. Deng, E. W. Hagley, J. Wen, R. Lutwak, K. Helmerson, S. L. Rolston, and W. D. Phillips, Phys. Rev. Lett. 82, 871 (1999). 
[21] J. Stenger, S. Inouye, A. P. Chikkatur, D. M. Stamper-Kurn, D. E. Pritchard, and W. Ketterle, Phys. Rev. Lett. 82, 4569 (1999).

[22] B. Desruelle, V. Boyer, P. Bouyer, G. Birkl, M. Lécrivain, F. Alves, C. Westbrook, and A. Aspect, Eur. Phys. J. D 1, 255 (1998).

[23] S. Richard, F. Gerbier, J. H. Thywissen, M. Hugbart, P. Bouyer, and A. Aspect, Phys. Rev. Lett. 91, 010405 (2003).

[24] I. Shvarchuck, C. Buggle, D. S. Petrov, K. Dieckmann, M. Zielonkovski, M. Kemmann, T. Tiecke, W. von Klitzing, G. V. Shlyapnikov, and J. T. M. Walraven, Phys. Rev. Lett. 89, 270404 (2002).

[25] W. Ketterle, D. S. Durfee, and D. M. Stamper-Kurn, in Proceedings of the International School of Physics "Enrico Fermi," edited by M. Inguscio, S. Stringari, and C. E. Wieman (IOS Press, Amsterdam, 1999), p. 67.

[26] The probe laser wavelength is $\lambda_{\mathrm{L}}=780.2 \mathrm{~nm}$; its intensity is $0.17 \mathrm{~mW} / \mathrm{cm}^{2}$, and the imaging pulse length is $34 \mu \mathrm{s}$. The halfwidth of the absorption resonance has been measured to be 8 $\mathrm{MHz}$, slightly broader than the natural linewidth $6 \mathrm{MHz}$. This could explain the value of the measured absorption cross section, found to be smaller than the resonant cross section $\sigma_{0}$ $=3 \lambda_{\mathrm{L}}^{2} / 4$ by a factor of $4.00(15)$ [27].

[27] F. Gerbier, J. H. Thywissen, S. Richard, M. Hugbart, P. Bouyer, and A. Aspect, Phys. Rev. Lett. 92, 030405 (2004).

[28] A possible cause for this behavior is that for our parameters, the radial expansion is already asymptotic $\left(\omega_{\perp} t \approx 57.0\right)$, whereas the axial expansion is not $\left(\omega_{z} t \approx 1.2\right)$. Thus, the spatial distribution along $z$ might be intermediate between the in-trap distribution and the asympotic one. Note however that using the temperature measured along the axial direction (with the same exclusion region than the one chosen to find $T_{x}$ ) would merely shift the temperature downwards by approximately
$5-10 \%$, without significantly affecting the main conclusions of the paper.

[29] F. Ferlaino, P. Maddaloni, S. Burger, F. S. Cataliotti, C. Fort, M. Modugno, and M.Inguscio, Phys. Rev. A 66, 011604(R) (2002).

[30] A. P. Chikkatur, A. Görlitz, D. M. Stamper-Kurn, S. Inouye, S. Gupta, and W. Ketterle, Phys. Rev. Lett. 85, 483 (2000).

[31] F. Zambelli, L. Pitaevskii, D. M. Stamper-Kurn, and S. Stringari, Phys. Rev. A 61, 063608 (2000).

[32] S. Giorgini, L. P. Pitaevskii, and S. Stringari, Phys. Rev. A 54, R4633 (1996).

[33] S. Giorgini, L. P. Pitaevskii, and S. Stringari, Phys. Rev. Lett. 78, 3987 (1997).

[34] This property holds in the semiclassical approximation, independently of making the HF approximation.

[35] Note also that integration along the probe line of sight decreases the visibility of the hole, even in the trap.

[36] P. Pedri, D. Guéry-Odelin, and S. Stringari, Phys. Rev. A 68, 043608 (2003).

[37] In addition, particle transfer is also possible [38,41], leading to a dynamic depletion of the condensate in the initial stages of the expansion.

[38] T. Nikuni and A. Griffin, Phys. Rev. A 65, 011601 (2002).

[39] Y. Castin and R. Dum, Phys. Rev. Lett. 77, 5315 (1996).

[40] Y. Kagan, E. L. Surkov, and G. V. Shlyapnikov, Phys. Rev. A 55, R18 (1997).

[41] E. Zaremba, T. Nikuni, and A. Griffin, J. Low Temp. Phys. 43, 141 (1999).

[42] F. Dalfovo, S. Giorgini, M. Guilleumas, L. Pitaevskii, and S. Stringari, Phys. Rev. A 56, 3840 (1997).

[43] W. Krauth, Phys. Rev. Lett. 77, 3695 (1996).

[44] M. Holzmann, W. Kratuh, and M. Narachewski, Phys. Rev. A 59, 2956 (1999). 